\title{
Simultaneous sequence transfer into two independent locations of a reporter vector using MultiSite Gateway ${ }^{\circledR}$ Technology
}

\author{
Julie Tubb ${ }^{1}$, Amy C. Groth ${ }^{1}$, Louis Leong ${ }^{2}$, and David W. Emery ${ }^{1}$
}

BioTechniques 39:553-557 (October 2005)

doi 10.2144/000112030

The bacteriophage $\lambda$ recombination system is increasingly used for recombinant DNA applications that involve the frequent transfer of sequences into and between shuttle and reporter vectors. This approach bypasses the need for restriction endonucleases or ligases and, as such, is easily scalable and automated. However, this system has not yet been tested for the ability to support the simultaneous introduction of donor fragments into two separate target sites of a single reporter plasmid. This attribute would greatly facilitate studies of cis-regulatory elements that only function in specific combinations, such as a class of regulatory elements known as chromatin insulators. With the goal of facilitating a screen for chromatin insulators, we sought to determine whether the commercially available MultiSite Gateway ${ }^{\circledR}$ Technology recombination system could be used to simultaneously insert candidate insulator elements into two separate locations of a functional reporter plasmid. We show that this application is both highly efficient and specific, generating the desired recombination products nearly three quarters of the time without disrupting the specificity of the reporter system. As such, these studies establish a novel application of the MultiSite Gateway Technology for the generation of recombinant reporter plasmids where the constituent elements function in a combinatorial fashion.

\section{INTRODUCTION}

Site-specific recombination based on the bacteriophage $\lambda$ recombination system is increasingly being used for high-throughput cloning, functional screens, and myriad other recombinant DNA applications (1-7). This approach offers the ability to easily clone and transfer specific donor sequences of interest into and between multiple target vectors without the use of restriction enzymes or ligases and, as such, is easily scalable and automated. Several forms of this technology, marketed under the Gateway ${ }^{\circledR}$ brand name, are commercially available through Invitrogen (Carlsbad, CA, USA). In its simplest form, this technology involves the recombination between a destination plasmid that contains the $\lambda$ att $\mathrm{P}$ or at $\mathrm{R}$ recombination target sites and either a PCR fragment or donor plasmid in which a sequence of interest is flanked with the cognate $a t t \mathrm{~B}$ or $a t t \mathrm{~L}$ recombination donor sequences $(1,2)$. The recombination reaction is mediated by a mixture of the $\lambda$ Int and $I H F$ proteins alone [for the att $\mathrm{B}-a t t \mathrm{P}$ reaction, marketed as $\mathrm{BP}$ Clonase ${ }^{\mathrm{TM}}$ (Invitrogen)] or in combination with the Xis protein [for the att $\mathrm{L}-a t t \mathrm{R}$ reaction, marketed as LR Clonase ${ }^{\mathrm{TM}}$ (Invitrogen)]. These recombination reactions are characterized by a high degree of efficiency and specificity. By employing positive and negative selection strategies, it is possible to assure that the desired recombinants are recovered at a rate approaching
$100 \%$ for individual recombination reactions. In a more complex permutation marketed as the MultiSite Gateway ${ }^{\circledR}$ Technology, hybrid att sites have been generated that allow for a wider range of recombination partners, while maintaining the high degree of efficiency and specificity characteristic of the simpler permutations (3-5).

However, to the best of our knowledge, this system has not yet been tested for the ability to support the simultaneous introduction of PCR-derived donor fragments into two separate target sites of a single destination reporter plasmid. We were interested in pursuing this ability in support of our efforts to screen candidate sequences for chromatin insulator activity. As previously reviewed $(8,9)$, chromatin insulators are naturally occurring DNA elements found in a diverse range of species that function as boundary elements to separate differentially regulated chromosomal loci. These elements function to block the effects of regulatory elements in adjacent chromosomal domains, including the activating effects of enhancers and/or the repressive effects of heterochromatin. Several lines of evidence suggest that, at least in some settings, insulators work best when used in pairs to flank genetic loci or reporter genes (8-11). Thus, any plasmidbased screens of chromatin insulator elements require that the candidate elements be inserted at two separate locations within the reporter plasmid. This paradigm could also be applied to other cis-regulatory elements where it is desirable

${ }^{1}$ University of Washington, Division of Medical Genetics, Seattle, WA and ${ }^{2}$ Invitrogen Corporation, Carlsbad, CA, USA 
to test combinations of candidate elements within single reporter constructs.

In order to determine whether the MultiSite Gateway Technology could be used for this purpose, we introduced selected att $\mathrm{P} / \mathrm{R}$ target sites into a previously described enhancerblocking insulator reporter plasmid diagramed in Figure 1 (12) and carried out recombination reactions with a variety of PCR fragments flanked by the cognate $a t t \mathrm{~B}$ recombination partners. Here we report a comprehensive analysis of the recombination products generated with this system. The data demonstrate that this application of the MultiSite Gateway Technology recombination system is both highly efficient and specific, generating the desired recombination products nearly three quarters of the time.

\section{MATERIALS AND METHODS}

\section{Vector Construction}

We started with the previously described Neo reporter plasmid pJC5-4 (12). This construct contains an expression

A
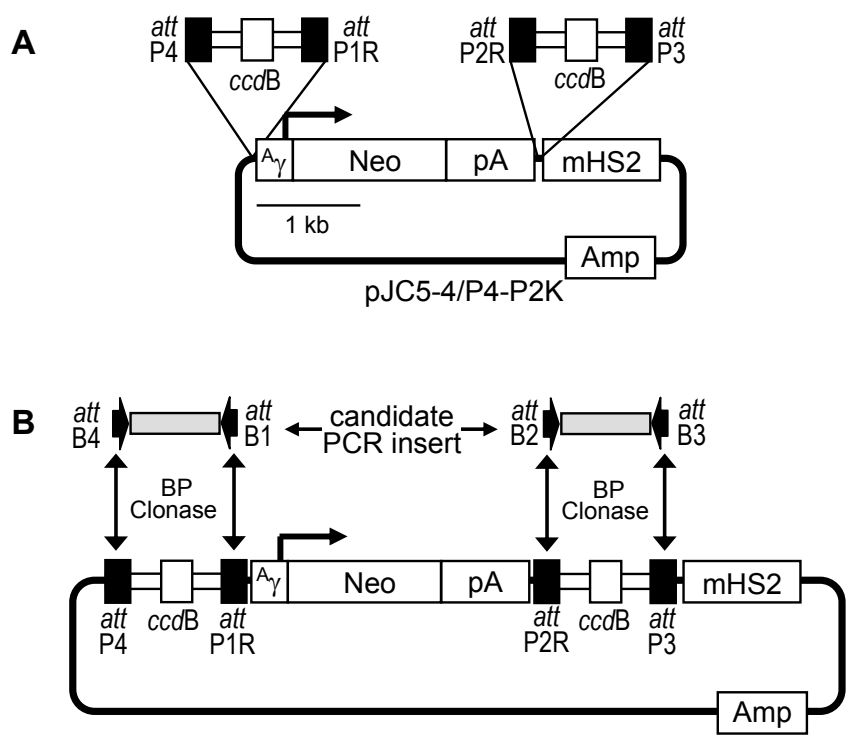

Figure 1. Experimental strategy. (A) Construction of reporter plasmid pJC5-4/P4-P2K: the original reporter plasmid (pJC5-4) contains a mammalian expression cassette for $\mathrm{NeO}$ composed of a promoter from the human A $\gamma$-globin gene, a coding cassette from TN5 Neo, and a transcript termination and polyadenylation signal from simian virus 40 (SV40), as well as an mHS2 enhancer (12). To this plasmid, we added two separate recombination cassettes (top) comprising distinct hybrid att $\mathrm{P} / \mathrm{R}$ recognition sequences flanking a negative selectable marker $c c d \mathrm{~B}$ : $a t t \mathrm{P} 4-c c d \mathrm{~B}-a t t \mathrm{P} 1 \mathrm{R}\left(5^{\prime}\right.$ of the ${ }^{\mathrm{A}} \gamma$ promoter) and $a t t \mathrm{P} 2 \mathrm{R}-c c d \mathrm{~B}-a t t \mathrm{P} 3$ ( $5^{\prime}$ of the mHS2 enhancer). (B) Exchange reactions. Matched sets of insert sequences (top) were prepared by PCR using hybrid primers (arrows) that introduce either a combination of attB4-attB1 or at $\mathrm{B} 2-a t \mathrm{~B} 3$ recognition sequences at the $5^{\prime}$ and $3^{\prime}$ ends of the PCR product, respectively. Recombination reactions mediated by BP Clonase result in the specific recombination between $a t \mathrm{~B}$ sequences on the donor fragments and $a t t \mathrm{P} / \mathrm{R}$ sequences on the destination reporter plasmid pJC5-4/P4-P2K as indicated, resulting in the simultaneous replacement of the $c c d \mathrm{~B}$ cassettes with the candidate inserts.
Table 1. attB PCR Amplification Primers

attB4 5'-GGGGACAACTTTGTATAGAAAAGTTG..(insert forward, $n=20$ )

t'B1 -GGGGACTGCTTTTTTGTACAAACTTG..(insert reverse, $n=20$ )

5'-GGGGACAGCTTTCTTGTACAAAGTGG..(insert forward, $n=20$ ) 5'-GGGGACAACTTTGTATAATAAAGTTG..(insert reverse, $n=20$ )

cassette for Neo composed of a promoter from the human ${ }^{\mathrm{A}} \gamma$ globin gene, a coding cassette from TN5 Neo, and a transcript termination and polyadenylation signal from simian virus 40 (SV40), all inserted into the BamHI site of the multiple cloning site of plasmid pGEM-4Z. This construct also contains an enhancer encompassing DNase I hypersensitive site 2 of the mouse $\beta$-globin locus control region (mHS2) inserted into the EcoRI site of pGEM-4Z. As diagramed in Figure $1 \mathrm{~A}$, this construct was modified by inserting $\operatorname{att} \mathrm{P} / \mathrm{R}$ target sites $5^{\prime}$ and $3^{\prime}$ of the Neo expression cassette to generate reporter plasmid pJC5-4/P4-P2K. For this purpose, we first deleted the chloramphenicol resistance $\left(\mathrm{Cm}^{\mathrm{r}}\right)$ gene (as a $B s a \mathrm{~A} 1-B b v \mathrm{C} 1$ fragment) from the Gateway cloning plasmids pDONRP4-P1R and pDONRP2R-P3 (Invitrogen) to reduce the size of the target sequence. The remaining attP4- $c c d \mathrm{~B}$ $a t t \mathrm{P} 1 \mathrm{R}$ and $a t t \mathrm{P} 2 \mathrm{R}-c c d \mathrm{~B}-a t t \mathrm{P} 3$ fragments were then excised as 1.4-kb NotI-AflII fragments and inserted in the $X b a \mathrm{I}$ and $K p n I$ sites of pJC5-4, respectively, after filling in the requisite overhangs. This essentially replaced the $1.2-\mathrm{kb}$ fragments located at these sites in the original pJC5-4 plasmid that contained the prototypic chromatin insulator DNase I hypersensitive site 4 of the chicken $\beta$-globin locus control region (cHS4). The resulting plasmid, pJC5-4/P4-P2K, and all other intermediates containing the negative selectable marker $c c d \mathrm{~B}$, were cloned in $c c d \mathrm{~B}$-resistant DB3.1 ${ }^{\mathrm{TM}}$ bacteria (Invitrogen) under ampicillin selection.

\section{Insert Fragments}

Candidate insert fragments were prepared from human genomic DNA by either a two-step or one-step PCR method. In the two-step method, one round of PCR was performed using approximately 21 nucleotide primers specific for the desired sequence, the resulting fragment was gel-purified, and a second round of PCR was performed using hybrid primers consisting of the sequences used in the original primers linked to specific att $\mathrm{B}$ donor sequences. As diagramed in Figure 1B and specified in Table 1, these included one PCR with primers containing att $\mathrm{B} 4$ and $a t t \mathrm{~B} 1$ and a separate $\mathrm{PCR}$ with primers containing at $\mathrm{B} 2$ and $a t \mathrm{~B} 3$. In the one-step method, a single round of PCR was performed on human genomic DNA directly using the hybrid primers described above. All final products were gel-purified and quantified by spectrophotometry.

\section{Exchange Reactions}

Exchange reactions were carried out following the manufacturer's instructions. In short, this entailed incubating 
the target plasmid pJC5-4/P4-P2K (150 ng) and matched sets of attB-flanked PCR products (100 ng each) with BP Clonase for $1 \mathrm{~h}$ at $25^{\circ} \mathrm{C}$. Aliquots were then used to transform $c c d \mathrm{~B}$-sensitive $\mathrm{DH} 5$ bacteria, and $c c d \mathrm{~B}$-negative, ampicillinresistant clones were isolated for further characterization.

\section{Functional Insulator Assay}

Exchange reactions were carried out to introduce fragments for either the prototypic chromatin insulator $\mathrm{cHS} 4$ or, as a negative control, the bacterial drug resistance gene Zeo, into the reporter plasmid pJC5-4/P4-P2K. Using previously described methods (12), the efficiency of reporter plasmid expression was then determined by transfecting the human erythroid cell line K562 and assaying for the frequency of colony formation in agarose cultures under G418 selection.

\section{RESULTS AND DISCUSSION}

In order to assess the ability of the MultiSite Gateway Technology to support the simultaneous introduction of donor fragments into two separate target sites of a destination reporter plasmid, we used the scheme diagramed in Figure 1. For the destination plasmid, we modified the previously described enhancer-blocking insulator reporter plasmid pJC5-4 (12) by introducing two distinct MultiSite Gateway Technology target sequences at separate locations to generate plasmid pJC5-4/P4-P2K. The target site located $5^{\prime}$ of the ${ }^{\mathrm{A}} \gamma$-globin gene promoter is comprised of att $\mathrm{P} 4$ and $a t t \mathrm{P} 1 \mathrm{R}$ sites flanking the negative selectable marker $c c d \mathrm{~B}$. The target site located between the polyadenylation and mHS2

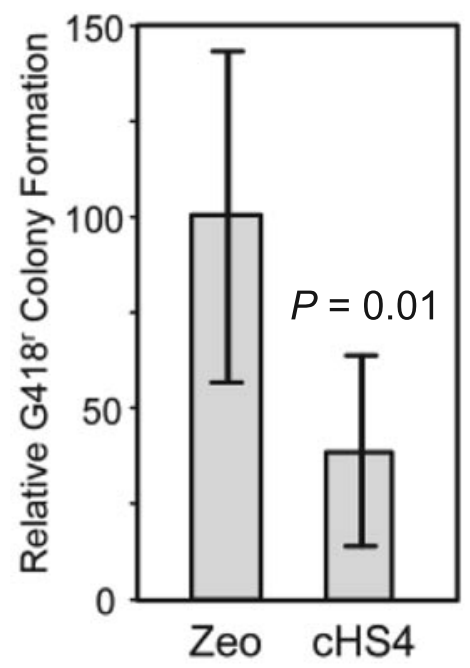

Figure 2. Functional insulator assay. The destination reporter plasmid pJC5-4/P4-P2K containing either the prototypic chromatin insulator cHS4 or, as a negative control, the bacterial drug resistance gene Zeo, was transfected into the human erythroid cell line K562 as previously described (12). The frequency of reporter plasmid expression was determined by measuring the frequency of colony formation under G418 selection. The results are the average \pm SD from 7 replicates performed in 3 separate experiments, and are reported as a percentage of the average colony formation obtained with the Zeo control within individual experiments. sequences is comprimised of matched $\operatorname{att} \mathrm{P} 2 \mathrm{R}$ and $\operatorname{att} \mathrm{P} 3$ sites flanking a second copy of $c c d \mathrm{~B}$.

As indicated at the top of Table 2, we tested 8 different candidate donor sequences, ranging in size from 453-876 bp. The candidate donor fragments were chosen based on the presence of sequence motifs similar to those found in the cHS4 prototypic chromatin insulator $(10,12)$, including a high $\mathrm{GC}$ content and extended poly $(\mathrm{G})$ tracks. These fragments were prepared by PCR using primers that added either a combination of att $\mathrm{B} 4$ and $a t t \mathrm{~B} 1$ or $a t t \mathrm{~B} 2$ and $a t t \mathrm{~B} 3$ sites to their $5^{\prime}$ and $3^{\prime}$ ends, respectively. In some cases, a two-step approach was employed in which the target sequences were first amplified from human genomic DNA with sequencespecific primers, and the resulting PCR product was used for a second round of amplification with hybrid primers consisting of the sequences used in the original primers linked to specific $a t t \mathrm{~B}$ donor sequences. In other cases, the target sequences were amplified directly with the hybrid primers. The primer sequences are presented in Table 1. We also included two control fragments amplified from plasmid DNA, including a 590-bp fragment containing a bacterial drug resistance gene, Zeo, and a previously described $1200-b p$ fragment containing the cHS4 chromatin insulator (12).

Exchange reactions were carried out with BP Clonase following the manufacturer's instructions. As diagramed in Figure 1B, each reaction included a combination of the destination reporter plasmid and matched pairs of attB4-attB1 and $a t t \mathrm{~B} 2-a t t \mathrm{~B} 3$ flanked donor fragments. Transfected bacteria were selected for the presence of $A m p^{r}$ and the absence of $c c d \mathrm{~B}$, and a total of 50 individual colonies (3-9 for each donor fragment) were chosen for further analysis. This included gross restriction mapping and direct sequencing using primers flanking the sites of recombination.

As summarized in Table 2, the recombination reactions were both highly efficient and specific, with the isolation of recombinant products containing donor fragments at both target sites of the destination reporter plasmid at a rate of $74 \%$ (37 of 50 products tested). This compares favorably to the overall rate of about $90 \%$ for single recombination reactions with PCR-derived fragments reported previously (1). The most predominant source of failure arose from the lack of insertion at one or both target sites in the destination reporter plasmid, accounting for approximately $10 \%$ (5 of 50) and 4\% (2 of 50) of the total events, respectively. Restriction mapping and direct sequencing indicated that, in these cases, the $c c d \mathrm{~B}$ cassettes within the nontargeted sites had been deleted by illegitimate recombination between the flanking attP/R sequences. In addition, 6 of the 50 recombination products analyzed (12\%) appeared to contain one copy of the donor fragment integrated by illegitimate recombination with the att $\mathrm{P} 4$ and att $\mathrm{P} 3$ target sites on the destination reporter plasmid, resulting in the deletion of the intervening ${ }^{A} \gamma$-Neo expression cassette. These cases of illegitimate recombination presumably reflect the low-level promiscuity of the $a t \mathrm{t} \mathrm{P} / \mathrm{att} \mathrm{B}$ recombination reaction. Moreover, recovered products containing illegitimate recombinations could be easily identified by diagnostic restriction mapping or PCR, providing a ready means of identifying and eliminating such products prior to further analysis. 
Table 2. Success Rate for Recombinase Reaction

\begin{tabular}{|c|c|c|c|c|c|c|c|c|c|c|c|}
\hline $\begin{array}{l}\text { Fragment Source } \\
\text { Fragment size (bp) }\end{array}$ & \multicolumn{8}{|c|}{ Genomic Fragments ${ }^{a}$} & $\begin{array}{c}\text { Zeo }^{\text {b }} \\
590\end{array}$ & \multicolumn{2}{|l|}{$\mathrm{cHS}^{\mathrm{b}}$} \\
\hline P4-P1R + P2R-P3 & 2 & 5 & 0 & 9 & 3 & 4 & 4 & 4 & 2 & 4 & $37(74 \%)$ \\
\hline P4-P1R only & 0 & 0 & 0 & 0 & 0 & 0 & 0 & 0 & 0 & 0 & $0(0 \%)$ \\
\hline P2R-P3 only & 0 & 2 & 0 & 0 & 0 & 3 & 0 & 0 & 0 & 0 & $5(10 \%)$ \\
\hline P4-P3 deletion & 3 & 0 & 1 & 0 & 1 & 0 & 0 & 0 & 1 & 0 & $6(12 \%)$ \\
\hline Total analyzed & 5 & 7 & 4 & 9 & 4 & 7 & 4 & 4 & 3 & 4 & $50(100 \%)$ \\
\hline
\end{tabular}

Table 3. PCR-Mediated Error Rate

\begin{tabular}{|c|c|c|c|}
\hline & & $\begin{array}{c}\text { Two-Step } \\
\text { PCR }\end{array}$ & $\begin{array}{c}\text { One-Step } \\
\text { PCR }\end{array}$ \\
\hline \multicolumn{2}{|c|}{ Correct Inserts } & $8(28 \%)$ & $19(50 \%)$ \\
\hline \multirow[t]{3}{*}{ Errors } & point mutations ${ }^{a}$ & $14(48 \%)$ & $17(45 \%)$ \\
\hline & $\operatorname{poly}(G)^{b}$ & $3(10 \%)$ & $0(0 \%)$ \\
\hline & both & $4(14 \%)$ & $2(5 \%)$ \\
\hline \multicolumn{4}{|c|}{$\begin{array}{l}\text { aOne or more point mutations or small deletions/insertions. } \\
\text { bOne or more deletions within poly }(G) \text { tracts. }\end{array}$} \\
\hline
\end{tabular}

As outlined in Table 3, we also detected a small but significant level of point mutations and small deletions in the donor fragments amplified from genomic DNA. For example, $72 \%$ of donor fragments generated by the two-step PCR method contained at least one point mutation or small deletion/insertion, a contraction of the $\operatorname{poly}(\mathrm{G})$ tract, or both. The frequency of such errors was less with the one-step PCR method but still averaged 50\%. This represents an overall mutation rate of approximately 1 in $750 \mathrm{bp}$, which compares favorably with the rates of PCR errors observed by others (13). This is especially true due to the $\operatorname{poly}(\mathrm{G})$ tracts in the donor fragments used for this study. It is important to keep in mind that the overall rate of such errors would be less with smaller fragments or fragments that do not contain poly $(\mathrm{G})$ tracts. It is also important to note that this source of error is unique to the use of PCR fragments as the source of donor sequences; no such errors would be anticipated if the donor fragments are provided in the form of plasmid, although other considerations involving the stoichiometry or topology of the participating fragments may reduce the general efficiency or accuracy of the recombination reaction under this scenario.

We can thus calculate, based on the more robust onestep PCR method, an overall rate for the production of desired constructs containing no point mutations as follows: 0.74 (rate of correct double recombination) $\times 0.50$ (rate of error-free PCR donor fragment in first site) $\times 0.5$ (rate of error-free PCR donor fragment in second site) $=0.19$. This indicates that nearly 1 in 5 recovered recombination products generated by this method can be expected to contain the desired double inserts that are free of PCR errors. We have confirmed these results with two other destination plasmids: one in which the reporter gene Neo was replaced with green fluorescent protein (GFP) and another where the att $\mathrm{P} 2 \mathrm{R}-$ att $\mathrm{P} 3$ recombination cassette was moved to the opposite side of the mHS2 enhancer.

To determine whether the presence of residual sequences from the $a t t \mathrm{P} / \mathrm{B}$ recombination junctions interfered with the functionality of the reporter plasmid, we transfected K562 cells with versions of the reporter plasmid carrying either the prototypic cHS4 chromatin insulator or, as a negative control, Zeo. As seen in Figure 2, inclusion of the cHS4 insulator significantly reduced the frequency of $\mathrm{G} 418^{\mathrm{r}}$ colony formation by $61 \%$ compared with the Zeo control $(P=0.01)$. These results are consistent with the results reported with the original reporter plasmid pJC5-4 (12) and serve to demonstrate that the inclusion of MultiSite Gateway Technology sequences in this reporter plasmid did not disrupt its functional specificity.

In conclusion, we have shown that the MultiSite Gateway Technology recombinase system can be used to efficiently and accurately introduce PCR-derived donor fragments into two different target sites of a destination reporter plasmid simultaneously, with the recovery of the desired dual recombinants nearly three quarters of the time. It is worth noting that this system can presumably be adapted for the simultaneous insertion of nonidentical donor sequences, or the insertion of candidate sequences in alternative orientations, within any appropriately engineered destination reporter plasmid. As such, our results provide a useful paradigm for the application of the MultiSite Gateway Technology to the study of multiple cis-regulatory elements where it is desirable to test such elements in pair-wise combinations.

\section{ACKNOWLEDGMENTS}

We thank Gary Felsenfeld for the pJC5-4 reporter plasmid and Mari Aker for technical advice and helpful discussions. This work was supported in part by grants from the National Institutes of Health. 


\section{COMPETING INTERESTS STATEMENT}

L.L. is an employee of Invitrogen Corporation, the manufacturer and distributor of the MultiSite Gateway Technology. The other authors declare no competing interests.

\section{REFERENCES}

1. Walhout, A.J., G.F. Temple, M.A. Brasch, J.L. Hartley, M.A. Lorson, S. van den Heuvel, and M. Vidal. 2000. GATEWAY recombinational cloning: application to the cloning of large numbers of open reading frames or ORFeomes. Methods Enzymol. 328:575-592.

2. Hartley, J.L., G.F. Temple, and M.A. Brasch. 2000. DNA cloning using in vitro site-specific recombination. Genome Res. 10:1788-1795.

3. Sasaki, Y., T. Sone, S. Yoshida, K. Yahata, J. Hotta, J.D. Chesnut, T. Honda, and F. Imamoto. 2004. Evidence for high specificity and efficiency of multiple recombination signals in mixed DNA cloning by the Multisite Gateway system. J. Biotechnol. 107:233-243.

4. Cheo, D.L., S.A. Titus, D.R. Byrd, J.L. Hartley, G.F. Temple, and M.A. Brasch. 2004. Concerted assembly and cloning of multiple DNA segments using in vitro site-specific recombination: functional analysis of multi-segment expression clones. Genome Res. 14:2111-2120.

5. Hope, I.A., J. Stevens, A. Garner, J. Hayes, D.L. Cheo, M.A. Brasch, and M. Vidal. 2004. Feasibility of genome-scale construction of promoter::reporter gene fusions for expression in Caenorhabditis elegans using a multisite gateway recombination system. Genome Res. 14:2070-2075.

6. Marsischky, G. and J. LaBaer. 2004. Many paths to many clones: a comparative look at high-throughput cloning methods. Genome Res. 14:2020-2028.

7. Stanyon, C.A., T. Limjindaporn, and R.L. Finley. 2003. Simultaneous cloning of open reading frames into several different expression vectors. BioTechniques 35:520-526.

8. West, A.G., M. Gaszner, and G. Felsenfeld. 2002. Insulators: many functions, many mechanisms. Genes Dev. 16:271-288.
9. Emery, D.W., M. Aker, and G. Stamatoyannopoulos. 2003. Chromatin insulators and position effects, p. 381-395. In S.C. Makrides (Ed.), Gene Transfer and Expression in Mammalian Cells. EIC Laboratories, Norwood, MA.

10.West, A.G., S. Huang, M. Gaszner, M.D. Litt, and G. Felsenfeld. 2004 Recruitment of histone modifications by USF proteins at a vertebrate barrier element. Mol. Cell 16:453-463.

11.Yannaki, E., J. Tubb, M. Aker, G. Stamatoyannopoulos, and D.W. Emery. 2002. Topological constraints governing the use of the chicken HS4 chromatin insulator in oncoretrovirus vectors. Mol. Ther. 5:589598.

12.Chung, J.H., M. Whiteley, and G. Felsenfeld. 1993. A $5^{\prime}$ element of the chicken beta-globin domain serves as an insulator in human erythroid cells and protects against position effect in Drosophila. Cell 74:505-514.

13.Xiong, A.S., Q.H. Yao, R.H. Peng, X. Li, H.Q. Fan, Z.M. Cheng, and Y. Li. 2004. A simple, rapid, high-fidelity and cost-effective PCR-based two-step DNA synthesis method for long gene sequences. Nucleic Acids Res. 32:e98.

Address correspondence to David W. Emery, Box 357720, HSB K236F, 1705 NE Pacific Street, University of Washington, Seattle, WA 98195-7720, USA. e-mail: demery@u.washington.edu

\section{To purchase reprints}

of this article, contact

Reprints@BioTechniques.com 